\title{
The impact of new modes of electronic communication in the treatment of severe acute respiratory syndrome coronavirus 2 infection
}

\author{
Zhaoxiong Fang ${ }^{1 \#}$, Zhiqiang Gu ${ }^{1 \#}$, Tian Zhang ${ }^{2 \#}$, Junjie Lei ${ }^{3}$, Lu Lin ${ }^{1}$, Zhixiang Yan ${ }^{4}$ Keke Feng ${ }^{5}$, \\ Mengsha Xie ${ }^{6}$, Shuanshuan $\mathrm{Guo}^{2}$, Zhigang Liu ${ }^{2}$, Zhongsi Hong ${ }^{7},{\mathrm{Xiaofeng} \mathrm{Li}^{1}}^{1}$ \\ ${ }^{1}$ Department of Gastroenterology, the Fifth Affiliated Hospital, Sun Yat-sen University, Zhuhai, China; ${ }^{2}$ Department of Oncology, the Fifth Affiliated \\ Hospital, Sun Yat-sen University, Zhuhai, China; ${ }^{3}$ Department of Neurology, the Fifth Affiliated Hospital, Sun Yat-sen University, Zhuhai, China; \\ ${ }^{4}$ Guangdong Provincial Key Laboratory of Biomedical Imaging and Guangdong Provincial Engineering Research Center of Molecular Imaging, the \\ Fifth Affiliated Hospital, Sun Yat-sen University, Zhuhai, China; ${ }^{5}$ Department of Rehabilitation Medicine, the Fifth Affiliated Hospital, Sun Yat- \\ sen University, Zhuhai, China; ${ }^{6}$ Department of Gerontology, the Fifth Affiliated Hospital, Sun Yat-sen University, Zhuhai, China; ${ }^{7}$ Department of \\ Infectious Disease, the Fifth Affiliated Hospital, Sun Yat-sen University, Zhuhai, China \\ Contributions: (I) Conception and design: X Li, Z Hong; (II) Administrative support: None; (III) Provision of study materials or patients: None; \\ (IV) Collection and assembly of data: All authors; (V) Data analysis and interpretation: All authors; (VI) Manuscript writing: All authors; (VII) Final \\ approval of manuscript: All authors. \\ \#These authors contributed equally to this work. \\ Correspondence to: Dr. Xiaofeng Li. Department of Gastroenterology, the Fifth Affiliated Hospital, Sun Yat-sen University, Zhuhai, China. \\ Email: zdwylxf@163.com; Dr. Zhongsi Hong. Department of Infectious Disease, the Fifth Affiliated Hospital, Sun Yat-sen University, Zhuhai, \\ China. Email: hongzhs@mail.sysu.edu.cn; Dr. Zhigang Liu. The Cancer Center of the Fifth Affiliated Hospital of Sun Yat-sen University, Zhuhai, \\ China. Email: Zhigangliu1983@hotmail.com.
}

Background: More than 26,760,000 cases of SARS-CoV-2 infection have been reported globally to date. This study aimed to analyze the impact of new electronic communication tools in the diagnosis and treatment of patients with SARS-CoV-2 infection.

Methods: From January 20 to February 26, 2020, adult patients with laboratory-confirmed severe acute respiratory syndrome coronavirus 2 (SARS-CoV-2) infection who were treated in The Fifth Affiliated Hospital, Sun Yat-sen University, in Zhuhai, China, were recruited. Forty-seven eligible patients were enrolled and randomly classified into either the test group or the control group. All of the patients received the standard therapeutic regimen and routine ward rounds. The test group was subdivided into three subgroups: the first subgroup (5-minute group) was given an extra 5-minute ward round by WeChat voice call once daily for basic disease communication; the second subgroup (10-minute group) received an extra 10-minute ward round by WeChat voice call once daily for further detail; and the third subgroup (20-minute group) was given an extra 10-minute ward round via WeChat voice call once daily, as well as an extra 10 minutes every 3 days. The primary outcome was the duration of positive-to-negative conversion of SARSCoV-2 nucleic acid diagnosed by the NAT (nucleic acid testing).

Results: In the test groups, the median time from diagnosis to the endpoint was 7.0 days [interquartile range (IQR), 3.8-10.8], compared with 10.0 days (IQR, 6.5-14.5) in the control group. It showed significant reduced the duration time of virus from positive to negative by the NAT (nucleic acid testing), $(\mathrm{P}=0.032)$ especially between the 10-minute subgroup (3.0 days; IQR, 3.0-7.5) and the control group $(\mathrm{P}=0.0065)$.

Conclusions: The use of new modes of electronic communication can benefit patients during the COVID-19 pandemic and could be extremely valuable in addressing the shortage of medical protective equipment and reducing occupational risk of exposure to infection.

Keywords: Coronavirus disease 2019 (COVID-19); severe acute respiratory syndrome coronavirus 2 infection (SARS-CoV-2 infection); electronic communication tool; therapy; physician-patient communication 
Submitted Aug 14, 2020. Accepted for publication Oct 05, 2020.

doi: $10.21037 /$ atm-20-6413

View this article at: http://dx.doi.org/10.21037/atm-20-6413

\section{Introduction}

As of September 5, 2020, coronavirus disease 2019 (COVID-19) had infected more than 26,760,000 people worldwide, resulting in 875,000 deaths $(1,2)$. Highthroughput sequencing has revealed that the virus that causes COVID-19, severe acute respiratory syndrome coronavirus 2 (SARS-CoV-2), shares more than $79 \%$ of its genomic sequence with severe acute respiratory syndrome coronavirus (SARS-CoV), a member of the subgenus Sarbecovirus (Beta-CoV lineage B) (3,4). Evidence pointing to the virus spread by person-to-person respiratory transmission and contact transmission rapidly. Information regarding the epidemiological and clinical features of COVID-19 is sparse (5). A small number of patients with severe SARS-CoV-2 infections go on to develop pneumonia, pulmonary edema, acute respiratory distress syndrome (ARDS), or in extremely severe cases, multiple organ failure or even death (6). Because of the global shortage of medical resources, we designed a new model of physician-patient communication, that will reduce the consumption of protective resources, as well as the risk of cross-infection of healthcare workers and patients, urgently needs to be discovered.

In this retrospective analysis, we aimed to improve working efficiency and reduce occupational exposure risk for medical personnel in the diagnosis and treatment of SARS-CoV-2 infection.

We present the following article in accordance with the CONSORT reporting checklist (available at http://dx.doi. org/10.21037/ atm-20-6413).

\section{Methods}

\section{Study design and participants}

Patients with SARS-CoV-2 infection who were treated in the Department of Infectious Disease in the Fifth Affiliated Hospital, Sun Yat-sen University, in Zhuhai, China, were enrolled between January 20 and February 26, 2020. The patients were diagnosed with SARS-CoV-2 infection according to the interim guidance from the World Health Organization (WHO) (3). The inclusion criteria were as follows: (I) aged 18-65 years old; (II) positive SARS-CoV-2 ribonucleic acid (RNA); (III) willing to provide respiratory swabs; and (IV) a WeChat user. Patients meeting any of the following criteria were excluded: (I) confirmed with severe SARS-CoV-2-causing pneumonia; (II) rapid deterioration to a severe condition; (III) complicated with malignant tumors or a compromised immune system; or (IV) unable to make daily voice calls via WeChat.

A confirmed case of SARS-CoV-2 infection was defined as a positive result from a high-throughput sequencing or realtime reverse transcription-polymerase chain reaction (RTPCR) assay of nasal or pharyngeal swab specimens $(1,7)$. The incubation period was defined as the duration from the date of contact with the source of transmission to the onset of symptoms. Respiratory samples were collected every 1-2 days until two sequential negative results were obtained.

The study was approved by the Medical Ethical Committee of the Fifth Affiliated Hospital of Sun Yat-sen University (approval No. L076-1) and informed consent was obtained from each participant. The study conformed to the provisions of the Declaration of Helsinki (as revised in 2013).

The patients were divided into the test group and the control group. Both groups received standard therapy and routine ward rounds, according to the guidelines of diagnosis and treatment of SARS-CoV-2 infection (standard version) (8). Any changes in the patients' conditions were monitored, recorded, and promptly addressed.

With the aim of exploring new modes of electronic communication in the treatment of infectious diseases, the test group patients were divided into three subgroups and given additional high-quality online ward rounds and psychological care via WeChat voice calls.

The first test subgroup (the 5-minute group) was given an extra online ward round via WeChat voice call for 5 minutes once a day. During these 5 -minute calls, patients could communicate with the doctor in charge about the disease progression of themselves. The second test subgroup (the 10-minute group) was given an extra online ward round via WeChat voice call for 10 minutes once a day. During these 10-minute calls, patients could communicate with the doctor in charge about the disease progression of themselves, as well as the extent and future outlook of the disease. The third test subgroup (the 20 
minutes group) was given the same treatment as the second group, with an additional 10 minutes every 3 days, which enabled the patients to get more detail information of disease. The fourth group given only standard therapy as the blank control group. Respecting patient consent, all patients were free to choose consent or opting-out.

\section{Data collection}

The epidemiological characteristics (including recent exposure history), clinical symptoms and signs, and laboratory findings of the patients were extracted from the hospital's electronic medical records. Computed tomography was used for radiologic assessment. Laboratory assessments included complete blood count, blood chemistry, coagulation test, liver and renal function, electrolytes, reactive protein, procalcitonin, lactate dehydrogenase, and creatine kinase. All data were checked by two physicians ( $\mathrm{ZF}$ and $\mathrm{ZG}$ ) and any differences in interpretation between the two primary reviewers were adjudicated by a third researcher (JL).

\section{Primary outcome}

The primary endpoint was the duration of positive-tonegative conversion of SARS-CoV-2 nucleic acid diagnosed by the NAT (nucleic acid testing). It is the time from laboratory conformed positive to negative of SARS-CoV-2 RNA result after two sequential negative respiratory tract sample results. The interval between the two negative results needed to be more than 24 hours.

\section{Statistical analysis}

SPSS (version 19.0) was used to perform all statistical analyses. Continuous variables were presented as median [interquartile range (IQR)] and compared by Mann-Whitney $\mathrm{U}$ test. Categorical variables were presented as number (\%) and compared by chi-square or Fisher's exact test.

\section{Results}

A total of 47 patients with laboratory-confirmed SARSCoV-2 infection who met the eligibility criteria and completed the baseline assessment were enrolled in the trial. As shown in Table 1, the demographics, clinical characteristics, and radiographic findings of the patients were similar between the test group and the control group on admission. There were 21 males and 26 females enrolled, with a median age of 36.0 years (IQR, 29.0-44.0). The median incubation period was 6.0 days (IQR, 2.0-10.0).

The duration of positive-to-negative conversion of SARS-CoV-2 RNA of the patients was tracked by viral NAT (Figure 1). The median time of the primary endpoint for test group was 7.0 days (IQR, 3.8-10.8), compared with 10.0 days (IQR, 6.5-14.5) in the control group $(\mathrm{P}=0.032)$ (Table 2). It showed significant reduced the duration time of virus from positive to negative by the NAT (nucleic acid testing), between the 10-minute subgroup (3.0 days; IQR, 3.0-7.5) and the control group $(\mathrm{P}=0.0065)$. The median conversion time was 7.0 days (IQR, 5.5 to 13.0) in the 5-minute group compared with 8.5 days (IQR, 4.8 to $16.0)$ in the 10 minutes +10 minutes group; no statistical difference existed between these groups and the control group $(\mathrm{P}=0.45$ and 0.56 , respectively).

\section{Discussion}

Previous studies have incorporated communication tools into standard medical consultation to improve patients' self-monitoring and understanding of disease $(9,10)$. The WeChat-based mobile health (mHealth) intervention via WeChat, which is the most popular social media app in china, was significantly reduced depressive symptoms among people living with HIV and depression (11). In our study, WeChat-based online ward rounds provided a contactless and effective channel for patient-doctor communication in the treatment of COVID-19. Voice calls were chosen due to them having higher efficiency than texting, as well as offering a higher level of privacy protection than video calls.

For the 47 patients in our study, the duration time of virus from positive to negative of sputum specimens ranged from 3.0-32.0 days. With SARS-CoV, viral RNA could be detected in the respiratory specimens of approximately onethird of patients for up to 4 weeks after disease onset (10). Similarly, Middle East respiratory syndrome coronavirus (MERS-CoV) RNA can be detected in lower respiratory specimens for at least 3 weeks after disease onset $(12,13)$. We found that remote doctor-patient communication could help to reduce the duration of positive-to-negative conversion of SARS-CoV-2 RNA; in particular, the 10-minute group had an advantage over other groups, with the duration of positive-to-negative conversion of viral RNA significantly reduced compared to the control group $(\mathrm{P}=0.032)$. High-quality communication and timely feedback were presumed to be the mechanisms underlying 
Table 1 Demographic, clinical, and radiographic characteristics of 47 patients with SARS-CoV-2 infection on admission

\begin{tabular}{|c|c|c|c|c|}
\hline Variable & All patients $(n=47)$ & Test group $(n=14)$ & Control group $(n=33)$ & $P$ value \\
\hline Age, years & $36.0(29.0-44.0)$ & $33.0(28.8-45.8)$ & $36.0(30.5-44.5)$ & 0.40 \\
\hline Male & $21(44.7)$ & $7(50.0)$ & $14(42.4)$ & 0.93 \\
\hline \multicolumn{5}{|l|}{ Comorbidity } \\
\hline Diabetes & $1(2.1)$ & $1(7.1)$ & 0 & 0.33 \\
\hline Hypertension & $2(4.3)$ & $2(14.3)$ & 0 & 0.10 \\
\hline Coronary heart disease & 0 & 0 & 0 & \\
\hline Cerebral vascular disease & 0 & 0 & 0 & \\
\hline Incubation period & $6.0(2.0-10.0)$ & $4.0(1.0-11.3)$ & $6.0(3.0-9.0)$ & 0.40 \\
\hline \multicolumn{5}{|l|}{ Signs and symptoms } \\
\hline Fever & $26(55.3)$ & $9(64.3)$ & $17(51.5)$ & 0.53 \\
\hline Cough & $11(23.4)$ & $2(14.3)$ & $9(27.3)$ & 0.46 \\
\hline Phlegm & $15(31.9)$ & $4(28.6)$ & $11(33.3)$ & 1.00 \\
\hline Hemoptysis & 0 & 0 & 0 & \\
\hline Conjunctiva injection & $1(2.1)$ & 0 & $1(3.0)$ & 1.00 \\
\hline Muscular soreness & $4(8.5)$ & 0 & $4(12.1)$ & 0.30 \\
\hline Sore throat & $9(19.1)$ & $2(14.3)$ & $7(21.2)$ & 0.70 \\
\hline Nausea and vomiting & $2(4.3)$ & 0 & $2(6.1)$ & 1.00 \\
\hline CT manifestation & & & & 0.52 \\
\hline Viral pneumonia & $29(61.7)$ & $10(71.4)$ & $19(57.6)$ & \\
\hline Non-viral pneumonia & $18(38.3)$ & $4(28.6)$ & $14(42.4)$ & \\
\hline $\begin{array}{l}\text { Loss of leukocytes/lymphocytes in blood } \\
\text { routine examination }\end{array}$ & $7(14.9)$ & $3(21.4)$ & $4(12.1)$ & 0.41 \\
\hline
\end{tabular}

Data are presented as median (interquartile range) and $\mathrm{n}(\%)$.

the good prognosis of these patients.

Furthermore, this new model of physician-patient communication by Wechat could be used to supplement standard therapy at minimal cost and could be easily popularized; it would also help to reduce the risk of occupational exposure and create safer working conditions for medical staff.

As the world continues to battle the COVID-19 


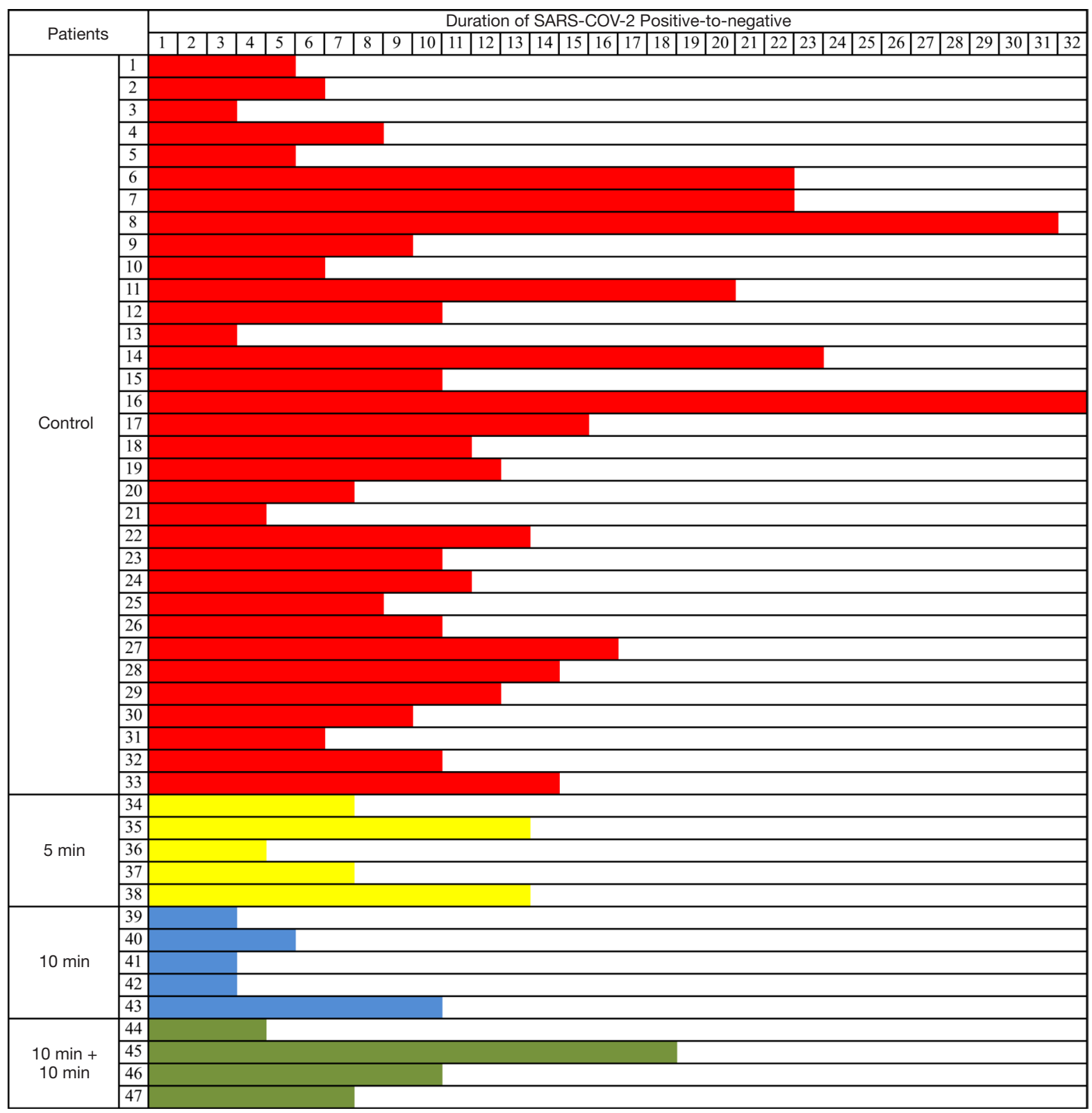

Figure 1 The duration of positive-to-negative conversion of SARS-CoV-2 RNA of 47 patients. This was defined as the time from a laboratory-confirmed positive SARS-CoV-2 RNA result to a laboratory-confirmed negative SARS-CoV-2 RNA result after two consecutive negative respiratory tract sample results. The interval between the two negative results must be more than 24 hours.

pandemic, medical resources could be badly needed and in short supply. The shortage of medical protection resources and the risk of occupational exposure facing medical personnel urgently need to be addressed (14). Our new mode of electronic communication could help to alleviate the demand for medical protective resources and assist with the evaluation of therapeutic effect, which indicates its potential value in the treatment of SARS-CoV-2 infection.
Our new electronic communication mode, which can ease up the shortage of medical resources and evaluate therapeutic effect, indicates its potential value in the treatment of SARS-CoV-2 infection.

\section{Conclusions}

The duration of positive-to-negative conversion of viral 
Table 2 The duration of positive-to-negative conversion of SARS$\mathrm{CoV}-2$ among the groups

\begin{tabular}{lll}
\hline Group & Duration of PTN & P value \\
\hline Control (vs.) & $10.0(6.5-14.5)$ & \\
Test group & $7.0(3.8-10.8)$ & 0.032 \\
5-min group & $7.0(5.5-13.0)$ & 0.45 \\
10-min group & $3.0(3.0-7.5)$ & 0.0065 \\
10-min + 10-min group & $8.5(4.8-16.0)$ & 0.56 \\
5-min group (vs.) & $7.0(5.5-13.0)$ & \\
10-min group & $3.0(3.0-7.5)$ & 0.064 \\
10-min + 10-min group & $8.5(4.8-16.0)$ & $>0.99$ \\
10-min group (vs.) & $3.0(3.0-7.5)$ & \\
10-min + 10-min group & $8.5(4.8-16.0)$ & 0.12 \\
\hline
\end{tabular}

Data are presented as median (interquartile range). PTN, positive-to negative.

RNA in the test group was significantly shorter than that of the control group. Our findings suggested that patients with SARS-CoV-2 infection might benefit from the use of this new mode of electronic communication, which could prove to be extremely valuable in reducing the shortage of medical protective resources and the risk of occupational exposure to infection.

\section{Acknowledgments}

We thank all patients involved in the study and all medical staff involved in the fight against COVID-19.

Funding: This work was supported by the National Key Research and Development Program of China (2020YFC082400), the Task-Force Project on the Prevention and Control of Novel Coronavirus of Guangdong Province (2020B11113001), the Three Major Constructions of Sun Yat-sen University (the TaskForce Project on the Prevention and Control of Novel Coronavirus of Sun Yat-sen University), the Emergency Task-Force Project on the Prevention and Control of Novel Coronavirus of Zhuhai 2020, the Guangzhou Laboratory of regenerative medicine and health.

\section{Footnote}

Reporting Checklist: The authors have completed the CONSORT reporting checklist. Available at http://dx.doi. org/10.21037/ atm-20-6413

Data Sharing Statement: Available at http://dx.doi. org/10.21037/ atm-20-6413

Conflicts of Interest: All authors have completed the ICMJE uniform disclosure form (available at http://dx.doi. org/10.21037/ atm-20-6413). The authors have no conflicts of interest to declare.

Ethical Statement: The authors are accountable for all aspects of the work in ensuring that questions related to the accuracy or integrity of any part of the work are appropriately investigated and resolved. The study was approved by the Medical Ethical Committee of the Fifth Affiliated Hospital of Sun Yat-sen University (approval No. L076-1) and informed consent was obtained from each participant. The study conformed to the provisions of the Declaration of Helsinki (as revised in 2013).

Open Access Statement: This is an Open Access article distributed in accordance with the Creative Commons Attribution-NonCommercial-NoDerivs 4.0 International License (CC BY-NC-ND 4.0), which permits the noncommercial replication and distribution of the article with the strict proviso that no changes or edits are made and the original work is properly cited (including links to both the formal publication through the relevant DOI and the license). See: https://creativecommons.org/licenses/by-nc-nd/4.0/.

\section{References}

1. Huang C, Wang Y, Li X, et al. Clinical features of patients infected with 2019 novel coronavirus in Wuhan, China. Lancet 2020;395:497-506.

2. Callaway E, Cyranoski D, Mallapaty S, et al. The coronavirus pandemic in five powerful charts. Nature 2020;579:482-3.

3. Lu R, Zhao X, Li J, et al. Genomic characterisation and epidemiology of 2019 novel coronavirus: implications for virus origins and receptor binding. Lancet 2020;395:565-74.

4. van Doremalen N, Bushmaker T, Morris DH, et al. Aerosol and surface stability of SARS-CoV-2 as compared with SARS-CoV-1. N Engl J Med 2020;382:1564-7.

5. Shimizu K. 2019-nCoV, fake news, and racism. Lancet 2020;395:685-6.

6. Wang D, Hu B, Hu C, et al. Clinical characteristics of 138 hospitalized patients with 2019 novel coronavirus-infected 
pneumonia in Wuhan, China. JAMA 2020;323:1061-9.

7. Chen L, Liu W, Zhang Q, et al. RNA based mNGS approach identifies a novel human coronavirus from two individual pneumonia cases in 2019 Wuhan outbreak. Emerg Microbes Infect 2020;9:313-9.

8. Jin YH, Cai L, Cheng ZS, et al. A rapid advice guideline for the diagnosis and treatment of 2019 novel coronavirus (2019-nCoV) infected pneumonia (standard version). Mil Med Res 2020;7:4.

9. Soh JY, Lee SU, Lee I, et al. A mobile phone-based selfmonitoring tool for perioperative gastric cancer patients with incentive spirometer: randomized controlled trial. JMIR Mhealth Uhealth 2019;7:e12204.

10. Xu D, Zhang Z, Jin L, et al. Persistent shedding of viable SARS-CoV in urine and stool of SARS patients during the convalescent phase. Eur J Clin Microbiol Infect Dis 2005;24:165-71.

Cite this article as: Fang Z, Gu Z, Zhang T, Lei J, Lin L, Yan Z, Feng K, Xie M, Guo S, Liu Z, Hong Z, Li X. The impact of new modes of electronic communication in the treatment of severe acute respiratory syndrome coronavirus 2 infection. Ann Transl Med 2020;8(19):1239. doi: 10.21037/atm-20-6413
11. Guo Y, Hong YA, Cai W, et al. Effect of a WeChat-based intervention (Run4Love) on depressive symptoms among people living with HIV in China: a randomized controlled trial. J Med Internet Res 2020;22:e16715.

12. Oh MD, Park WB, Choe PG, et al. Viral load kinetics of MERS coronavirus infection. N Engl J Med 2016;375:1303-5.

13. Corman VM, Albarrak AM, Omrani AS, et al. Viral shedding and antibody response in 37 patients with Middle East respiratory syndrome coronavirus infection. Clin Infect Dis 2016;62:477-83.

14. Liu Y, Li J, Feng Y. Critical care response to a hospital outbreak of the 2019-nCoV infection in Shenzhen, China. Crit Care 2020;24:56.

(English Language Editor: J. Reynolds) 